\title{
Article \\ Generalization of the Regularization Method to Singularly Perturbed Integro-Differential Systems of Equations with Rapidly Oscillating Inhomogeneity
}

\author{
Abdukhafiz Bobodzhanov ${ }^{1}$, Burkhan Kalimbetov ${ }^{2, *}$ and Valeriy Safonov ${ }^{1}$ \\ 1 Department of Higher Mathematics, National Research University "MPEI", Krasnokazarmennaya 14, \\ 111250 Moscow, Russia; bobojanova@yandex.ru (A.B.); Sinsaf@yandex.ru (V.S.) \\ 2 Department of Mathematics, Khoja Ahmet Yasawi International Kazakh-Turkish University, \\ B. Sattarkhanov 29, Turkestan 161200, Kazakhstan \\ * Correspondence: burkhan.kalimbetov@ayu.edu
}

check for updates

Citation: Bobodzhanov, A.; Kalimbetov, B.; Safonov, V. Generalization of the Regularization Method to Singularly Perturbed Integro-Differential Systems of Equations with Rapidly Oscillating Inhomogeneity. Axioms 2021, 10, 40. https://doi.org/10.3390/ axioms10010040

Academic Editor: Valery Y. Glizer and Florin Felix Nichita

Received: 18 January 2021

Accepted: 16 March 2021

Published: 22 March 2021

Publisher's Note: MDPI stays neutral with regard to jurisdictional claims in published maps and institutional affiliations.

Copyright: (c) 2021 by the authors. Licensee MDPI, Basel, Switzerland. This article is an open access article distributed under the terms and conditions of the Creative Commons Attribution (CC BY) license (https:// creativecommons.org/licenses/by/ $4.0 /)$.

\begin{abstract}
In this paper, we consider systems of singularly perturbed integro-differential equations with a rapidly oscillating right-hand side, including an integral operator with a slowly varying kernel. Differential equations of this type and integro-differential equations with slowly varying inhomogeneity and with a rapidly oscillating coefficient at an unknown function are studied. The main goal of this work is to generalize the Lomov's regularization method and to reveal the influence of the rapidly oscillating right-hand side on the asymptotics of the solution to the original problem.
\end{abstract}

Keywords: singular perturbation; integro-differential equation; regularization; rapidly oscillating inhomogeneity; asymptotic convergence; resonant exhibitors

MSC: 35R09; 45K05

\section{Introduction}

When studying various applied problems related to the properties of media with a period structure, it is necessary to study differential equations with rapidly oscillating irregularities. Equations of this type are often found, for example, in electrical tric systems under the influence of high-frequency external forces. The presence of such forces creates serious problems for the numerical integration of the corresponding differential equations. Therefore, asymptotic methods are usually applied to such equations, the most famous of which are the Feshchenko-Shkil-Nikolenko splitting method [1,2] and the Lomov's regularization method [3-14]. The splitting method is especially effective when applied to equations with a rapidly oscillating inhomogeneity, and in the case of an inhomogeneity containing both rapidly and slow components, the Lomov's regularization method turned out to be the most effective. However, both of these methods were developed mainly for singularly perturbed equations that do not contain an integral operator. The transition from differential equations to integro-differential equations requires a significant restructuring of the algorithm of the regularization method. The integral term generates new types of singularities in solutions that differ from the previously known ones, which complicates the development of the algorithm for the regularization method. As far as we know, the splitting method has not been applied to integro-differential equations.

In the present study, the Lomov's regularization method is generalized to previously not studied classes of problems such as

$$
L_{\varepsilon} y(t, \varepsilon) \equiv \varepsilon \frac{d y}{d t}-A(t) y-\int_{t_{0}}^{t} K(t, s) y(s, \varepsilon) d s=h_{1}(t)+h_{2}(t) e^{\frac{i \beta(t)}{\varepsilon}}, y\left(t_{0}, \varepsilon\right)=y^{0}, \quad t \in\left[t_{0}, T\right]
$$

where $y=\left\{y_{1}(t, \varepsilon), \ldots, y_{n}(t, \varepsilon)\right\}$ is an unknown vector function, $A(t)$ is $(n \times n)$-matrix, $h_{j}(t)=\left(h_{1 j}(t), \ldots, h_{n j}(t)\right)$ are known vector functions, $\beta^{\prime}(t)>0$ is the frequency of a rapidly 
oscillating inhomogeneity, $\varepsilon>0$ is a small parameter. Problem (1) will be considered under the following conditions:

(1) $A(t) \in C^{\infty}\left(\left[t_{0}, T\right], \mathbb{C}^{n^{2}}\right), h_{1}(t), h_{2}(t) \in C^{\infty}\left(\left[t_{0}, T\right], \mathbb{C}^{n}\right), K(t, s) \in C^{\infty}\left(\left\{t_{0} \leq s \leq t \leq T\right\}, \mathbb{C}^{n^{2}}\right)$;

(2) The spectrum $\left\{\lambda_{1}(t), \ldots, \lambda_{n}(t)\right\}$ of the matrix $A(t)$ and the frequency $\beta^{\prime}(t)$ satisfy for all $t \in\left[t_{0}, T\right]$ the requirements:

(a) $\quad \beta^{\prime}(t)>0, \quad \lambda_{i}(t) \neq \lambda_{j}(t), i \neq j, \lambda_{j}(t) \neq 0, \lambda_{j}(t) \neq i \beta^{\prime}(t), i, j=\overline{1, n}$;

(b) $\quad \operatorname{Re} \lambda_{j}(t) \leq 0, j=\overline{1, n}$.

Thus, problem (1) is considered (in the terminology of [3]) in the nonresonant case $\lambda_{j}(t) \neq i \beta^{\prime}(t), i, j=\overline{1, n}, \forall t \in\left[t_{0}, T\right]$. The resonant case $\lambda_{j}(t) \equiv i \beta^{\prime}(t), i, j=\overline{1, n}, \forall t \in$ $\left[t_{0}, T\right]$ it is supposed to be considered in our subsequent work.

Note that in [15], the scalar case of a singularly perturbed integro-differential equation with a rapidly oscillating factor for an unknown function was considered. In this paper, in contrast to [15], we investigate a more general case of a system of integro-differential equations, and the rapidly oscillating factor is a coefficient on the right-hand side, for one of its inhomogeneities.

\section{Regularization of the Problem (1)}

We denote $\lambda_{n+1}(t) \equiv i \beta^{\prime}(t)$ and introduce the regularizing variables

$$
\tau_{j}=\frac{1}{\varepsilon} \int_{t_{0}}^{t} \lambda_{j}(\theta) d \theta \equiv \frac{\psi_{j}(t)}{\varepsilon}, j=\overline{1, n+1}
$$

and instead of problem (1) consider the problem

$$
\begin{aligned}
& L_{\varepsilon} \tilde{y}(t, \tau, \varepsilon) \equiv \varepsilon \frac{\partial \tilde{y}}{\partial t}+\sum_{j=1}^{n+1} \lambda_{j}(t) \frac{\partial \tilde{y}}{\partial \tau_{j}}-A(t) \tilde{y}- \\
& -\int_{t_{0}}^{t} K(t, s) \tilde{y}\left(s, \frac{\psi(s)}{\varepsilon}, \varepsilon\right) d s=h_{1}(t)+h_{2}(t) e^{\tau_{n+1}} \sigma, \\
& \left.\tilde{y}(t, \tau, \varepsilon)\right|_{t=t_{0}, \tau=0}=y^{0}, t \in\left[t_{0}, T\right]
\end{aligned}
$$

for the function $\tilde{y}=\tilde{y}(t, \tau, \varepsilon)$, which is denoted $\tau=\left(\tau_{1}, \ldots, \tau_{n+1}\right), \psi=\left(\psi_{1}, \ldots, \psi_{n+1}\right)$, $\sigma=e^{\frac{i}{\varepsilon} \beta\left(t_{0}\right)}$.

It is clear that, if $\tilde{y}=\tilde{y}(t, \tau, \varepsilon)$ is the solution of the problem (2), then the vector function $y=\tilde{y}\left(t, \frac{\psi(t)}{\varepsilon}, \varepsilon\right)$ is an exact solution of the problem (1); therefore, the problem (2) is extended with respect to the problem (1). However, it cannot be considered completely regularized, since the integral term

$$
J \tilde{y} \equiv J\left(\left.\tilde{y}(t, \tau, \varepsilon)\right|_{t=s, \tau=\psi(s) / \varepsilon}\right)=\int_{t_{0}}^{t} K(t, s) \tilde{y}\left(s, \frac{\psi(s)}{\varepsilon}, \varepsilon\right) d s
$$

has not been regularized in it. To regularize it, we introduce a class $M_{\varepsilon}$ that is asymptotically invariant with respect to the operator $J \tilde{y}$ (see [3]; p. 62).

Let us first consider the space $U$ of vector functions $y(t, \tau, \sigma)$, represented by the sums

$$
y(t, \tau, \sigma)=y_{0}(t, \sigma)+\sum_{j=1}^{n+1} y_{j}(t, \sigma) e^{\tau_{j}}, \quad y_{j}(t, \sigma) \in C^{\infty}\left(\left[t_{0}, T\right], \mathbb{C}^{n}\right), j=\overline{0, n+1} .
$$

Note that in (3) elements of the space $U$ depend on bounded in $\varepsilon>0$ the constant $\sigma=\sigma(\varepsilon)$, which does not affect the development of the algorithm described below; therefore, henceforth, in the record of the element (3) of this space, for the sake of brevity, we omit the dependence on $\sigma$. 
Let us show that the class $M_{\varepsilon}=\left.U\right|_{\tau=\psi(t) / \varepsilon}$ is asymptotically invariant with respect to the operator $J$. The image of the operator $J$ on the element (3) of the space $U$ has the form:

$$
J y(t, \tau)=\int_{t_{0}}^{t} K(t, s) y_{0}(s) d s+\sum_{j=1}^{n+1} \int_{t_{0}}^{t} K(t, s) y_{j}(s) e^{\frac{1}{\varepsilon} \int_{t_{0}}^{s} \lambda_{j}(\theta) d \theta} d s .
$$

Integrating by parts, we will have

$$
\begin{gathered}
J_{j}(t, \varepsilon)=\int_{t_{0}}^{t} K(t, s) y_{j}(s) e^{\frac{1}{\varepsilon} \int_{t_{0}}^{s} \lambda_{j}(\theta) d \theta} d s= \\
=\varepsilon \int_{t_{0}}^{t} \frac{K(t, s) y_{j}(s)}{\lambda_{j}(s)} d e^{\frac{1}{\varepsilon} \int_{t_{0}}^{s} \lambda_{j}(\theta) d \theta}=\varepsilon\left[\left.\frac{K(t, s) y_{j}(s)}{\lambda_{j}(s)} e^{\frac{1}{\varepsilon} \int_{t_{0}}^{s} \lambda_{j}(\theta) d \theta}\right|_{s=t_{0}} ^{s=t}-\right. \\
\left.-\int_{t_{0}}^{t}\left(\frac{\partial}{\partial s} \frac{K(t, s) y_{j}(s)}{\lambda_{j}(s)}\right) e^{\frac{1}{\varepsilon} \int_{t_{0}}^{s} \lambda_{j}(\theta) d \theta} d s\right]=\varepsilon\left[\frac{K(t, t) y_{j}(t)}{\lambda_{j}(t)} e^{\frac{1}{\varepsilon} \int_{t_{0}}^{t} \lambda_{j}(\theta) d \theta}-\frac{K\left(t, t_{0}\right) y_{j}\left(t_{0}\right)}{\lambda_{j}\left(t_{0}\right)}\right]- \\
-\varepsilon \int_{t_{0}}^{t}\left(\frac{\partial}{\partial s} \frac{K(t, s) y_{j}(s)}{\lambda_{j}(s)}\right) e^{\frac{1}{\varepsilon} \int_{t_{0}}^{s} \lambda_{j}(\theta) d \theta} d s, j=\overline{1, n+1} .
\end{gathered}
$$

Continuing this process further, we obtain the expansion

$$
J_{j}(t, \varepsilon)=\sum_{j=1}^{n+1} \sum_{v=0}^{\infty}(-1)^{v} \varepsilon^{v+1}\left[\left(I_{j}^{v}\left(K(t, s) y_{j}(s)\right)\right)_{s=t} e^{\frac{1}{\varepsilon} \int_{t_{0}}^{t} \lambda_{j}(\theta) d \theta}-\left(I_{j}^{v}\left(K(t, s) y_{j}(s)\right)\right)_{s=t_{0}}\right]
$$

where the operators are introduced:

$$
I_{j}^{0}=\frac{1}{\lambda_{j}(s)} \cdot I_{j}^{v}=\frac{1}{\lambda_{j}(s)} \frac{\partial}{\partial s} I_{j}^{v-1}, j=\overline{1, n+1},(v \geq 1) .
$$

Hence, the image of the operator $J$ on an element (3) of the space $U$ can be represented as a series

$$
\begin{gathered}
J y(t, \varepsilon)=\int_{t_{0}}^{t} K(t, s) y_{0}(s) d s+ \\
+\sum_{j=1}^{n+1} \sum_{v=0}^{\infty}(-1)^{v} \varepsilon^{v+1}\left[\left(I_{j}^{v}\left(K(t, s) y_{j}(s)\right)\right)_{s=t} e^{\frac{1}{\varepsilon} \int_{t_{0}}^{t} \lambda_{j}(\theta) d \theta}-\left(I_{j}^{v}\left(K(t, s) y_{j}(s)\right)\right)_{s=t_{0}}\right] .
\end{gathered}
$$

It is easy to show (see, for example, [16], pp. 291-294) that this series converges asymptotically for $\varepsilon \rightarrow+0$ (uniformly in $t \in\left[t_{0}, T\right]$ ). This means that the class $M_{\varepsilon}$ is asymptotically invariant (for $\varepsilon \rightarrow+0$ ) with respect to the operator $J$.

Let us introduce operators $R_{v}: U \rightarrow U$, acting on each element $y(t, \tau) \in U$ of the form (3) according to the law:

$$
\begin{gathered}
R_{0} y(t, \tau)=\int_{t_{0}}^{t} K(t, s) y_{0}(s) d s, \\
R_{1} y(t, \tau)=\sum_{j=1}^{n+1}\left[\left(I_{j}^{0}\left(K(t, s) y_{j}(s)\right)\right)_{s=t} e^{\tau_{j}}-\left(I_{j}^{0}\left(K(t, s) y_{j}(s)\right)\right)_{s=t_{0}}\right], \\
R_{v+1} y(t, \tau)=\sum_{j=1}^{n+1} \sum_{v=0}^{\infty}(-1)^{v}\left[\left(I_{j}^{v}\left(K(t, s) y_{j}(s)\right)\right)_{s=t} e^{\tau_{j}}-\left(I_{j}^{v}\left(K(t, s) y_{j}(s)\right)\right)_{s=t_{0}}\right], v \geq 1 .
\end{gathered}
$$


Now let $\tilde{y}(t, \tau, \varepsilon)$ be an arbitrary continuous for $(t, \tau) \in\left[t_{0}, T\right] \times\left\{\tau: \operatorname{Re} \tau_{j} \leq 0, j=\overline{1, n+1}\right\}$ function, having an asymptotic expansion

$$
\tilde{y}(t, \tau, \varepsilon)=\sum_{k=0}^{\infty} \varepsilon^{k} y_{k}(t, \tau), \quad y_{k}(t, \tau) \in U
$$

converging when $\varepsilon \rightarrow+0$ (uniformly in $(t, \tau) \in\left[t_{0}, T\right] \times\left\{\tau: \operatorname{Re} \tau_{j} \leq 0, j=\overline{1, n+1}\right\}$ ). Then the image $J \tilde{y}(t, \tau, \varepsilon)$ of this function expands into an asymptotic series

$$
J \tilde{y}(t, \tau, \varepsilon)=\sum_{k=0}^{\infty} \varepsilon^{k} J y_{k}(t, \tau)=\left.\sum_{r=0}^{\infty} \varepsilon^{r} \sum_{s=0}^{r} R_{r-s} y_{s}(t, \tau)\right|_{\tau=\psi(t) / \varepsilon} .
$$

This equality is the basis for introducing an extension of the operator $J$ on series of the form (5):

$$
\tilde{J} \tilde{y}(t, \tau, \varepsilon) \equiv \tilde{J}\left(\sum_{k=0}^{\infty} \varepsilon^{k} y_{k}(t, \tau)\right) \stackrel{d e f}{=} \sum_{r=0}^{\infty} \varepsilon^{r} \sum_{s=0}^{r} R_{r-s} y_{s}(t, \tau) .
$$

Although the operator $\tilde{J}$ is defined formally, its usefulness is obvious, since in practice the $N$-th approximation of the asymptotic solution of problem (2) is usually constructed, in which only $\mathrm{N}$-th partial sums of the series (5) will participate, which have not formal but true meaning. Now we can write down the problem that is completely regularized with respect to the original problem (1):

$$
\begin{aligned}
L_{\varepsilon} \tilde{y}(t, \tau, \varepsilon) & \equiv \frac{\partial \tilde{y}}{\partial t}+\sum_{j=1}^{n+1} \lambda_{j}(t) \frac{\partial \tilde{y}}{\partial \tau_{j}}-A(t) \tilde{y}-\tilde{J} \tilde{y}=h_{1}(t)+ \\
& +\left.h_{2}(t) e^{\tau_{n+1} \sigma,} \tilde{y}(t, \tau, \varepsilon)\right|_{t=t_{0}, \tau=0}=y^{0}, \quad t \in\left[t_{0}, T\right] .
\end{aligned}
$$

\section{Iterative Problems and Their Solvability in the Space $U$}

Substituting the series (5) into (6) and equating the coefficients at the same degrees of $\varepsilon$, we obtain the following iterative problems:

$$
\begin{gathered}
L y_{0}(t, \tau) \equiv \sum_{j=1}^{n+1} \lambda_{j}(t) \frac{\partial y_{0}}{\partial \tau_{j}}-A(t) y_{0}-R_{0} y_{0}=h_{1}(t)+h_{2}(t) e^{\tau_{n+1}} \sigma, \quad y_{0}\left(t_{0}, 0\right)=y^{0} \\
L y_{1}(t, \tau)=-\frac{\partial y_{0}}{\partial t}+R_{1} y_{0}, \quad y_{1}\left(t_{0}, 0\right)=0 ; \\
L y_{2}(t, \tau)=-\frac{\partial y_{1}}{\partial t}+R_{1} y_{1}+R_{2} y_{0}, \quad y_{2}\left(t_{0}, 0\right)=0 ; \\
\ldots \\
L y_{k}(t, \tau)=-\frac{\partial y_{k-1}}{\partial t}+R_{k} y_{0}+\ldots+R_{1} y_{k-1}, \quad y_{k}\left(t_{0}, 0\right)=0, k \geq 1 .
\end{gathered}
$$

Each of the iterative problems $(7 \mathrm{~d})$ can be written as

$$
L y(t, \tau) \equiv \sum_{j=1}^{n+1} \lambda_{j}(t) \frac{\partial y}{\partial \tau_{j}}-A(t) y-R_{0} y=H(t, \tau), \quad y\left(t_{0}, 0\right)=y_{*}
$$

where $H(t, \tau)=H_{0}(t)+\sum_{j=1}^{n+1} H_{j}(t) e^{\tau_{j}}$ is the known function of the space $U$, and the operator $R_{0}$ has the form (see (4a)):

$$
R_{0} y \equiv R_{0}\left(y_{0}(t)+\sum_{j=1}^{n+1} y_{j}(t) e^{\tau_{j}}\right)=\int_{t_{0}}^{t} K(t, s) y_{0}(s) d s
$$


We introduce the scalar (for each $t \in\left[t_{0}, T\right]$ ) product in the space $U$ :

$$
\begin{aligned}
<z, w>\equiv & <z_{0}(t)+\sum_{j=1}^{n+1} z_{j}(t) e^{\tau_{j}}, w_{0}(t)+\sum_{j=1}^{n+1} w_{j}(t) e^{\tau_{j}}>\stackrel{\text { def }}{=} \\
& \stackrel{\text { def }}{=}\left(z_{0}(t), w_{0}(t)\right)+\sum_{j=1}^{n+1}\left(z_{j}(t), w_{j}(t)\right)
\end{aligned}
$$

where $(*, *)$ denotes the usual scalar product in a complex space $\mathbb{C}^{n}$. We prove the following statement.

Theorem 1. Let conditions (1), (2a) hold and the right-hand side $H(t, \tau)=H_{0}(t)+\sum_{j=1}^{n+1} H_{j}(t) e^{\tau_{j}}$ of the system (8) belongs to the space $U$. Then, for the solvability of system (8) in $U$ it is necessary and sufficient that the identities

$$
<H(t, \tau), \chi_{k}(t) e^{\tau_{k}}>\equiv 0, k=\overline{1, n}, \forall t \in\left[t_{0}, T\right]
$$

are carried out (here $\chi_{k}(t)$ are the eigenvectors of the conjugate matrix $A^{*}(t)$, corresponding to the eigenvalues $\bar{\lambda}_{j}(t), j=\overline{1, n}$.)

Proof. We will define the solution of the system (8) as an element (3) of the space $U$ :

$$
y(t, \tau)=y_{0}(t)+\sum_{j=1}^{n+1} y_{j}(t) e^{\tau_{j}}
$$

Substituting (10) into the system (8), we will have

$$
\sum_{j=1}^{n+1}\left[\lambda_{j}(t) I-A(t)\right] y_{j}(t) e^{\tau_{j}}-A(t) y_{0}(t)-\int_{t_{0}}^{t} K(t, s) y_{0}(s) d s=H_{0}(t)+\sum_{j=1}^{n+1} H_{j}(t) e^{\tau_{j}} .
$$

Equating here separately the free term and coefficients at the same exponents, we obtain the following equations:

$$
\begin{aligned}
& -A(t) y_{0}(t)-\int_{t_{0}}^{t} K(t, s) y_{0}(s) d s=H_{0}(t), \\
& {\left[\lambda_{j}(t) I-A(t)\right] y_{j}(t)=H_{j}(t), j=\overline{1, n+1 .}}
\end{aligned}
$$

Due to the smoothness of the kernel $-A^{-1}(t) K(t, s)$ and heterogeneity $-A^{-1}(t) H_{0}(t)$, the integral Equation (11a) has a unique solution $y_{0}(t) \in C^{\infty}\left(\left[t_{0}, T\right], \mathbb{C}^{n}\right)$. As $\lambda_{n+1}(t)=$ $i \beta^{\prime}(t)$ is not a point in the spectrum of the matrix $A(t)$ (see condition 2a)), then the Equation (11b) has a unique solution $y_{n+1}(t)=\left[\lambda_{n+1}(t) I-A(t)\right]^{-1} H_{n+1}(t)$ in the space $C^{\infty}\left(\left[t_{0}, T\right], \mathbb{C}^{n}\right)$.

Systems $(11 \mathrm{~b})$ for $j=1,2, \ldots, n$, are solvable in the space $C^{\infty}\left(\left[t_{0}, T\right], \mathbb{C}^{n}\right)$ if and only if the identities $\left(H_{j}(t), \chi_{j}(t)\right) \equiv 0, j=\overline{1, n}, \forall t \in\left[t_{0}, T\right]$ hold. It is easy to see that these identities coincide with the identities (9). Thus, the conditions (9) are necessary and sufficient for the solvability of the system (8) in the space $U$. The theorem is proved.

Remark 1. If the identities (9) hold, then under the conditions (1) and (2a) the system (8) has the following solution in the space $U$ :

$$
\begin{aligned}
y(t, \tau) & =y_{0}(t)+\sum_{j=1}^{n}\left[\alpha_{j}(t) \varphi_{j}(t)+\sum_{s=1, s \neq j}^{n} \frac{\left(H_{j}(t), \chi_{s}(t)\right)}{\lambda_{j}(t)-\lambda_{s}(t)} \varphi_{s}(t)\right] e^{\tau_{j}}+ \\
& +\left(\lambda_{n+1}(t) I-A(t)\right)^{-1} H_{n+1}(t) e^{\tau_{n+1}}
\end{aligned}
$$


where $\varphi_{k}(t)$ are eigenvectors of the matrix $A(t)$, corresponding to the eigenvalues $\lambda_{j}(t), j=$ $\overline{1, n},\left(\varphi_{k}(t), \chi_{j}(t)\right) \equiv \delta_{k j}, \alpha_{j}(t) \in C^{\infty}\left(\left[t_{0}, T\right], \mathbb{C}^{n}\right)$ are arbitrary scalar functions $j=\overline{1, n}, y_{0}(t)$ is the solution of the integral system (11a).

\section{Unique Solvability of the General Iterative Problem in the Space $U$ Remainder Theorem}

As seen from (12), the solution of the system (8) is determined ambiguously. However, if its solution satisfies to the additional conditions

$$
\begin{aligned}
& y\left(t_{0}, 0\right)=y_{* \prime} \\
& <-\frac{\partial y}{\partial t}+R_{1} y+Q(t, \tau), \chi_{k}(t) e^{\tau_{k}}>\equiv 0, k=\overline{1, n}, \forall t \in\left[t_{0}, T\right]
\end{aligned}
$$

where $Q(t, \tau)=Q_{0}(t)+\sum_{j=1}^{n+1} Q_{i}(t) e^{\tau_{j}}$ is a known function of the space $U, y_{*}$ is a constant number of the complex space $\mathbb{C}^{n}$, then system (8) will be uniquely solvable in the space $U$. More precisely, the following result holds.

Theorem 2. Let conditions (1), (2a) be satisfied, the right-hand side $H(t, \tau)$ of the system (8) belongs to the space $U$ and satisfies the orthogonality condition (9). Then system (8) under additional conditions (13a) is uniquely solvable in $U$.

Proof. Under condition (9), system (8) has a solution (12) in the space $U$, where the functions $\alpha_{j}(t) \in C^{\infty}\left(\left[t_{0}, T\right], \mathbb{C}^{n}\right), j=\overline{1, n}$, are still arbitrary. Subordinating (12) to the first condition (13a), i.e., $y\left(t_{0}, 0\right)=y_{*}$, we obtain the equation

$$
\sum_{j=1}^{n} \alpha_{j}\left(t_{0}\right) \varphi_{j}\left(t_{0}\right)=y^{*}
$$

where

$$
y^{*}=y_{*}+A^{-1}\left(t_{0}\right) H_{0}\left(t_{0}\right)-\sum_{j=1}^{n} \sum_{s=1, s \neq j}^{n} \frac{\left(H_{j}\left(t_{0}\right), \chi_{s}\left(t_{0}\right)\right)}{\lambda_{j}\left(t_{0}\right)-\lambda_{s}\left(t_{0}\right)} \varphi_{s}\left(t_{0}\right)-\left(\lambda_{n+1}\left(t_{0}\right) I-A\left(t_{0}\right)\right)^{-1} H_{n+1}\left(t_{0}\right) .
$$

Multiplying scalarly the equality (13b) by $\chi_{j}\left(t_{0}\right)$ and taking into account the biorthogonality of the systems $\left\{\varphi_{j}(t)\right\}$ and $\left\{\chi_{j}(t)\right\}$, we find the values

$$
\alpha_{j}\left(t_{0}\right)=\left(y^{*}, \chi_{j}\left(t_{0}\right)\right), j=\overline{1, n} .
$$

Let us now subordinate solution (12) to the second condition (13a). The right-hand side of this equation has the form

$$
\begin{aligned}
& -\frac{\partial y_{0}}{\partial t}+R_{1} y_{0}+Q(t, \tau)=-\dot{y}_{0}(t)-\sum_{j=1}^{n}\left[\left(\alpha_{j}(t) \varphi_{j}(t)\right)^{\bullet}+\sum_{s=1, s \neq j}^{n}\left(\frac{\left(H_{j}(t), \chi_{s}(t)\right)}{\lambda_{j}(t)-\lambda_{s}(t)} \varphi_{s}(t)\right)^{\bullet}\right]- \\
& -\left(\left(\lambda_{n+1}(t) I-A(t)\right)^{-1} H_{n+1}(t)\right) e^{\tau_{n+1}}+\sum_{j=1}^{n+1}\left[\frac{K(t, t) y_{j}(t)}{\lambda_{j}(t)} e^{\tau_{j}}-\frac{K\left(t, t_{0}\right) y_{j}\left(t_{0}\right)}{\lambda_{j}\left(t_{0}\right)}\right]+Q(t, \tau) .
\end{aligned}
$$

Now multiplying (15) scalarly by $\chi_{j}(t) e^{\tau_{j}}, j=\overline{1, n}$, we obtain equations

$$
\begin{gathered}
\dot{\alpha}_{j}(t)+\left(\dot{\varphi}_{j}(t)-\frac{K(t, t)}{\lambda_{j}(t)} \varphi_{j}(t), \chi_{j}(t)\right) \alpha_{j}(t)+ \\
+\sum_{s=1, s \neq j}^{n} \frac{\left(H_{j}(t), \chi_{s}(t)\right)}{\lambda_{j}(t)-\lambda_{s}(t)}\left(\dot{\varphi}_{s}(t), \chi_{j}(t)\right)+\left(Q_{j}(t), \chi_{j}(t)\right)=0, j=\overline{1, n} .
\end{gathered}
$$


Adding the initial conditions (14) to them, we can uniquely find the functions $\alpha_{j}(t)$, $j=\overline{1, n}$ :

$$
\begin{aligned}
& \alpha_{j}(t)=e^{-\int_{t_{0}}^{t}\left[\left(\dot{\varphi}_{j}(x)-\frac{K(x, x) \varphi_{j}(x)}{\lambda_{j}(x)}, \chi_{j}(x)\right)\right] d x}\left[\alpha_{j}\left(t_{0}\right)+\right. \\
& \left.+\int_{t_{0}}^{t} e^{\int_{t_{0}}^{s}\left[\left(\dot{\varphi}_{j}(x)-\frac{K(x, x) \varphi_{j}(x)}{\lambda_{j}(x)}, \chi_{j}(x)\right)\right] d x} l_{j}(s) d s\right], j=\overline{1, n}
\end{aligned}
$$

where $l_{j}(t)=-\left(Q_{j}(t), \chi_{j}(t)\right)-\sum_{s=1, s \neq j}^{n} \frac{\left(H_{j}(t), \chi_{s}(t)\right)}{\lambda_{j}(t)-\lambda_{s}(t)}\left(\dot{\varphi}_{s}(t), \chi_{j}(t)\right)$, and hence, we define the solution (12) of the system (8) in the space in a unique way. The theorem is proved.

Applying Theorems 1 and 2 to iterative problems (7d), we uniquely find their solutions in the space $U$ and construct series (5). Let $y_{\varepsilon N}(t)=\sum_{k=0}^{N} \varepsilon^{k} y_{k}\left(t, \frac{\varphi(t)}{\varepsilon}\right)$ is the restriction of the $N$-th partial sum of series (5) for $\tau=\frac{\varphi(t)}{\varepsilon}$. The same as in [3], it is easy to prove the following statement.

Lemma 1. Let conditions (1) and (2) be satisfied. Then the partial sum $y_{\varepsilon N}(t)$ satisfies problem (1) up to $O\left(\varepsilon^{N+1}\right)(\varepsilon \rightarrow+0)$, i.e.,

$$
\begin{aligned}
& \qquad \varepsilon \frac{d y_{\varepsilon N}(t)}{d t} \equiv A(t) y_{\varepsilon N}(t)+\int_{t_{0}}^{t} K(t, s) y_{\varepsilon N}(s) d s+h_{1}(t)+h_{2}(t) e^{\frac{i \beta(t)}{\varepsilon}} \sigma+\varepsilon^{N+1} R_{N}(t, \varepsilon), \\
& \qquad y_{\varepsilon N}\left(t_{0}\right)=y^{0}, \forall t \in\left[t_{0}, T\right] \\
& \text { where }\left\|R_{N}(t, \varepsilon)\right\|_{C\left[t_{0}, T\right]} \leq \bar{R}_{N} \text { for all } t \in\left[t_{0}, T\right] \text { and for all } \varepsilon \in\left(0, \varepsilon_{N}\right]
\end{aligned}
$$

Consider now the following problem:

$$
\varepsilon \frac{d z(t, \varepsilon)}{d t}=A(t) z(t, \varepsilon)+\int_{t_{0}}^{t} K(t, s) z(s, \varepsilon) d s+\Phi(t, \varepsilon), z\left(t_{0}, \varepsilon\right)=0, t \in\left[t_{0}, T\right] .
$$

Let us show that this problem is solvable in the space $C^{1}\left(\left[t_{0}, T\right], \mathbb{C}^{n}\right)$ (i.e., it has a solution for any right-hand side $\left.\Phi(t, \varepsilon) \in C\left(\left[t_{0}, T\right], \mathbb{C}^{n}\right)\right)$ and that in this case there is an estimate

$$
\|z(t, \varepsilon)\|_{C\left[t_{0}, T\right]} \leq \frac{v_{0}}{\varepsilon}\|\Phi(t, \varepsilon)\|_{C\left[t_{0}, T\right]} .
$$

Theorem 3. Let conditions (1) and (2) be satisfied. Then, for sufficiently small $\varepsilon \in\left(0, \varepsilon_{0}\right]$, problem (17) for any right-hand side $\Phi(t, \varepsilon) \in C\left[t_{0}, T\right]$ has a unique solution $z(t, \varepsilon)$ in the space $C^{1}\left(\left[t_{0}, T\right], \mathbb{C}^{n}\right)$ and estimate (18) holds, where $v_{0}$ is a constant independent of $\varepsilon>0$.

Proof. Introduce an additional unknown function

$$
u(t, \varepsilon)=\int_{t_{0}}^{t} K(t, s) z(s, \varepsilon) d s .
$$

Differentiating it with respect to $t$, we will have

$$
\frac{d u(t, \varepsilon)}{d t}=K(t, t) z(t, \varepsilon)+\int_{t_{0}}^{t} \frac{\partial K(t, s)}{\partial t} z(s, \varepsilon) d s .
$$

From this and (17) it follows that the vector function $w=\{z, u\}$ satisfies the following system:

$$
\begin{aligned}
& \varepsilon \frac{d w(t, \varepsilon)}{d t}=\left(\begin{array}{cc}
A(t) & I_{n} \\
0 & 0
\end{array}\right) w(t, \varepsilon)+\varepsilon\left(\begin{array}{c}
0 \\
K(t, t) z+\int_{t_{0}}^{t} \frac{\partial K(t, s)}{\partial t} z(s, \varepsilon) d s
\end{array}\right)+ \\
& +\left(\begin{array}{c}
\Phi(t, \varepsilon) \\
0
\end{array}\right), w\left(t_{0}, \varepsilon\right)=0 .
\end{aligned}
$$


Denote by $Y(t, s, \varepsilon)$ the normal fundamental matrix of the homogeneous system $\varepsilon \frac{d w}{d t}=\left(\begin{array}{cc}A(t) & I_{n} \\ 0 & 0\end{array}\right) w$, i.e., the matrix satisfying the equation

$$
\varepsilon \frac{d Y(t, s, \varepsilon)}{d t}=\left(\begin{array}{ll}
A(t) & I_{n} \\
0 & 0
\end{array}\right) Y(t, s, \varepsilon), Y(s, s, \varepsilon)=I, t_{0} \leq s \leq t \leq T .
$$

Since the matrix $\left(\begin{array}{cc}A(t) & I_{n} \\ 0 & 0\end{array}\right)$ is a matrix of simple structure and its spectrum $\left\{\lambda_{1}(t), \ldots, \lambda_{n}(t), 0\right\}$ lies in the half-plane $\operatorname{Re} \lambda(t) \leq 0$, then the Cauchy matrix $Y(t, s, \varepsilon)$ is uniformly bounded, i.e.,

$$
\|Y(t, s, \varepsilon)\| \leq c_{0} \forall(t, s, \varepsilon): t_{0} \leq s \leq t \leq T, \varepsilon>0
$$

where the constant $c_{0}>0$ does not depend on $\varepsilon>0$ (see, for example, [3], pp. 119-120). We now write down an integral system equivalent to system (19a):

$$
\begin{aligned}
& w(t, \varepsilon)=\int_{t_{0}}^{t} Y(t, \zeta, \varepsilon)\left(K(\zeta, \zeta) z(\zeta, \varepsilon)+\int_{t_{0}}^{\zeta \zeta} \frac{\partial K(\zeta, s)}{\partial t} z(s, \varepsilon) d s\right) d \zeta+ \\
& +\frac{1}{\varepsilon} \int_{t_{0}}^{t} Y(t, \zeta, \varepsilon)\left(\begin{array}{c}
\Phi(\zeta, \varepsilon) \\
0
\end{array}\right) d \zeta
\end{aligned}
$$

Since for each $\varepsilon>0$ there exists the solution $w(t, \varepsilon)$ of the system (19a) in the space $C^{1}\left(\left[t_{0}, T\right], \mathbb{C}^{\mathrm{n}}\right)$, then substituting it into $(19 \mathrm{~b})$, we obtain the identity. Let us move on to the norms:

$$
\begin{gathered}
\|w(t, \varepsilon)\| \leq \int_{t_{0}}^{t}\|Y(t, \zeta, \varepsilon)\| \cdot\|K(\zeta, \zeta)\| \cdot\|z(\zeta, \varepsilon)\| d \zeta+\int_{t_{0}}^{t}\|Y(t, \zeta, \varepsilon)\| \times \\
\times \int_{t_{0}}^{\zeta}\left\|\frac{\partial K(\zeta, s)}{\partial t}\right\| \cdot\|z(s, \varepsilon)\| d s d \zeta+\frac{1}{\varepsilon} \int_{t_{0}}^{t}\|Y(t, \zeta, \varepsilon)\| \cdot\|\Phi(\zeta, \varepsilon)\| d \zeta \leq \\
\leq c_{0} k_{0} \int_{t_{0}}^{t}\|w(\zeta, \varepsilon)\| d \zeta+c_{0} k_{1} \int_{t_{0}}^{t} \int_{t_{0}}^{\zeta}\|w(s, \varepsilon)\| d s d \zeta+ \\
\quad+\frac{T_{0}}{\varepsilon} c_{0}\|\Phi(t, \varepsilon)\| C\left[t_{0}, T\right] \leq c_{0} k_{0} \int_{t_{0}}^{t}\|w(s, \varepsilon)\| d s+ \\
+c_{0} k_{1} \int_{t_{0}}^{t} \int_{t_{0}}^{t}\|w(s, \varepsilon)\| d s d \zeta+\frac{c_{0} T_{0}}{\varepsilon}\|\Phi(t, \varepsilon)\|_{C\left[t_{0}, T\right]} \leq \\
\leq c_{0} k_{0} \int_{t_{0}}^{t}\|w(s, \varepsilon)\| d s+c_{0} k_{1} \int_{t_{0}}^{T} \int_{t_{0}}^{t}\|w(s, \varepsilon)\| d s d \zeta+ \\
+\frac{c_{0} T_{0}}{\varepsilon}\|\Phi(t, \varepsilon)\|_{C\left[t_{0}, T_{0}\right]} \leq\left(c_{0} k_{0}+c_{0} k_{1} T_{0}\right) \int_{t_{0}}^{t}\|w(s, \varepsilon)\| d s+ \\
+\frac{c_{0} T_{0}}{\varepsilon}\|\Phi(t, \varepsilon)\|_{C\left[t_{0}, T_{0}\right]}
\end{gathered}
$$

where $T_{0}=T-t_{0},\|K(t, s)\|_{C\left(\left[t_{0}, T\right] \times\left[t_{0}, T\right]\right)}=k_{0},\|\partial K(t, s) / \partial t\|_{C\left(\left[t_{0}, T\right] \times\left[t_{0}, T\right]\right)}=k_{1}$. We get the inequality

$$
\|w(t, \varepsilon)\| \leq \frac{c_{0} T_{0}}{\varepsilon}\|\Phi(t, \varepsilon)\|_{C\left[t_{0}, T_{0}\right]}+\left(c_{0} k_{0}+c_{0} k_{1} T_{0}\right) \int_{t_{0}}^{t}\|w(s, \varepsilon)\| d s .
$$


Applying the Gronwall-Bellman lemma to this inequality, we have

$$
\begin{gathered}
\|w(t, \varepsilon)\| \leq \frac{c_{0} T_{0}}{\varepsilon}\|\Phi(t, \varepsilon)\|_{C\left[t_{0}, T\right]} e^{\left(c_{0} k_{0}+c_{0} k_{1} T_{0}\right) \int_{t_{0}}^{t} d s .}= \\
=\frac{c_{0} T_{0}}{\varepsilon}\|\Phi(t, \varepsilon)\|_{C\left[t_{0}, T\right]} e^{\left(c_{0} k_{0}+c_{0} k_{1} T_{0}\right)\left(t-t_{0}\right)} \leq \frac{v_{0}}{\varepsilon}\|\Phi(t, \varepsilon)\|_{C\left[t_{0}, T\right]} \Rightarrow \\
\Rightarrow\|z(t, \varepsilon)\|_{C\left[t_{0}, T\right]} \leq \frac{v_{0}}{\varepsilon}\|\Phi(t, \varepsilon)\|_{C\left[t_{0}, T\right]}
\end{gathered}
$$

where $v_{0}=c_{0} T_{0} \cdot \max _{t \in\left[t_{0}, T\right]} e^{\left(c_{0} k_{0}+c_{0} k_{1} T_{0}\right)\left(t-t_{0}\right)}$. The theorem is proved.

Theorem 4. Let conditions (1) and (2) be satisfied. Then for any $\varepsilon \in\left(0, \varepsilon_{0}\right]$, where $\varepsilon_{0}>0$ is small enough, the problem (1) has a unique solution $y(t, \varepsilon) \in C^{1}\left(\left[t_{0}, T\right], \mathbb{C}^{\mathrm{n}}\right)$; in this case, the estimate

$$
\left\|y(t, \varepsilon)-y_{\varepsilon N}(t)\right\|_{C\left[t_{0}, T\right]} \leq C_{N} \varepsilon^{N+1}(N=0,1,2 \ldots)
$$

holds true, where the constant $C_{N}>0$ does not depend on $\varepsilon \in\left(0, \varepsilon_{0}\right]$.

Proof. By the lemma, the partial sum $y_{\varepsilon N}(t)$ satisfies the problem (16), so the remainder $r_{N}(t, \varepsilon) \equiv y(t, \varepsilon)-y_{\varepsilon N}(t)$ satisfies the following problem:

$$
\varepsilon \frac{r_{N}(t, \varepsilon)}{d t}=A(t) r_{N}(t, \varepsilon)+\int_{t_{0}}^{t} K(t, s) r_{N}(s, \varepsilon) d s+\varepsilon^{N+1} R_{N}(t, \varepsilon), r_{N}\left(t_{0}, \varepsilon\right)=0
$$

where $\Phi(t, \varepsilon)=-\varepsilon^{N+1} \int_{t_{0}}^{t} R_{N}(s, \varepsilon) d s$. By Theorem 3, we have the estimate

$$
\left\|r_{N}(t, \varepsilon)\right\|_{C\left[t_{0}, T\right]} \leq \varepsilon^{N} \bar{R}_{N}
$$

for all $N=0,1,2, \ldots$ and all $\varepsilon \in\left(0, \varepsilon_{N}\right]$, which means that the partial sum $y_{\varepsilon, N+1}(t)=$ $=y_{\varepsilon N}(t)+\varepsilon^{N+1} y_{N+1}\left(t, \frac{\psi(t)}{\varepsilon}\right)$ satisfies the inequality

$$
\left\|y(t, \varepsilon)-y_{\varepsilon, N+1}(t)\right\|_{C\left[t_{0}, T\right]} \equiv\left\|(y(t, \varepsilon)-y(t))-\varepsilon^{N+1} y_{N+1}\left(t, \frac{\psi(t)}{\varepsilon}\right)\right\|_{C\left[t_{0}, T\right]} \leq \bar{C}_{N+1} \varepsilon^{N+1} .
$$
have

Using the inequality $\|a-b\| \geq|||a||-\|b \mid\|$, valid for any numbers $a$ and $b$, we will

$$
\left\|y(t, \varepsilon)-y_{\varepsilon N}(t)\right\|_{C\left[t_{0}, T\right]} \leq\left(\bar{C}_{N}+\left\|y_{N+1}\left(t, \frac{\psi(t)}{\varepsilon}\right)\right\|_{C\left[t_{0}, T\right]}\right) \varepsilon^{N+1}
$$

whence we derive the estimate

$$
\left\|y(t, \varepsilon)-y_{\varepsilon N}(t)\right\|_{C\left[t_{0}, T\right]} \leq C_{N} \varepsilon^{N+1}
$$

where the constant $C_{N}>0$ does not depend on $\varepsilon \in\left(0, \varepsilon_{N}\right]$.

\section{Construction of the Solution of the First Iteration Problem in the Space $U$}

Using Theorem 1 , we will try to find a solution to the first iterative problem (7d). Since the right-hand side $h_{1}(t)+h_{2}(t) e^{\tau_{n+1}}$ of the system (7a), satisfies the condition (9), this system has (according to (12)) a solution in the space $U$ in the form

$$
y_{0}(t, \tau)=y_{0}^{(0)}(t)+\sum_{k=1}^{n} \alpha_{k}^{(0)}(t) \varphi_{k}(t) e^{\tau_{k}}+\left(\lambda_{n+1}(t) I-A(t)\right)^{-1} h_{2}(t) e^{\tau_{n+1}} \sigma
$$


where $\alpha_{k}^{(0)}(t) \in C^{\infty}\left(\left[t_{0}, T\right], \mathbb{C}^{n}\right)$ are arbitrary functions, $k=\overline{1, n}, y_{0}^{(0)}(t)$ is the solution of the integral system $-A(t) y_{0}(t)-\int_{t_{0}}^{t} K(t, s) y_{0}(s) d s=h_{1}(t)$.

Subordinating (20) to the initial condition $y_{0}\left(t_{0}, 0\right)=y^{0}$, we will have

$$
\begin{gathered}
y_{0}^{(0)}\left(t_{0}\right)+\sum_{k=1}^{n} \alpha_{k}^{(0)}\left(t_{0}\right) \varphi_{k}\left(t_{0}\right)+\left(\lambda_{n+1}\left(t_{0}\right) I-A\left(t_{0}\right)\right)^{-1} h_{2}\left(t_{0}\right) \sigma=y^{0} \Leftrightarrow \\
\Leftrightarrow \sum_{k=1}^{n} \alpha_{k}^{(0)}\left(t_{0}\right) \varphi_{k}\left(t_{0}\right)=y^{0}+A^{-1}\left(t_{0}\right) h_{1}\left(t_{0}\right)-\left(\lambda_{n+1}\left(t_{0}\right) I-A\left(t_{0}\right)\right)^{-1} h_{2}\left(t_{0}\right) \sigma .
\end{gathered}
$$

Now, scalarly multiplying this equality by $\chi_{j}\left(t_{0}\right), j=\overline{1, n}$, we obtain values

$$
\alpha_{k}^{(0)}\left(t_{0}\right)=\left(y^{0}+A^{-1}\left(t_{0}\right) h\left(t_{0}\right)-\left(\lambda_{n+1}\left(t_{0}\right) I-A\left(t_{0}\right)\right)^{-1} h_{2}\left(t_{0}\right) \sigma, \chi_{k}\left(t_{0}\right)\right), k=\overline{1, n} .
$$

For a complete calculation of the function $\alpha_{k}^{(0)}(t)$, we pass to the next iterative problem (7b). Substituting the solution (20) of the system (7a) into it, we arrive at the following equation:

$$
\begin{gathered}
L y_{1}(t, \tau)=-\frac{d}{d t} y_{0}^{(0)}(t)-\sum_{k=1}^{n} \frac{d}{d t}\left(\alpha_{k}^{(0)}(t) \varphi_{k}(t)\right) e^{\tau_{k}}- \\
-\frac{d}{d t}\left(\left(\lambda_{n+1}(t) I-A(t)\right)^{-1} h_{2}(t)\right) e^{\tau_{n+1}} \sigma+ \\
+\sum_{j=1}^{n}\left[\frac{\left(K(t, t) \alpha_{j}^{(0)}(t) \varphi_{j}(t)\right)}{\lambda_{j}(t)} e^{\tau_{j}}-\frac{\left(K\left(t, t_{0}\right) \alpha_{j}^{(0)}\left(t_{0}\right) \varphi_{j}\left(t_{0}\right)\right)}{\lambda_{j}\left(t_{0}\right)}\right] .
\end{gathered}
$$

Subordinating the right-hand side of this system to the solvability conditions (9), we obtain the system of ordinary differential equations

$$
-\frac{d \alpha_{k}^{(0)}(t)}{d t}+\left(\frac{K(t, t) \varphi_{k}(t)}{\lambda_{k}(t)}-\dot{\varphi}_{k}(t), \chi_{k}(t)\right) \alpha_{k}^{(0)}(t)=0, k=\overline{1, n} .
$$

Adding the initial condition (21) to them, we find

$$
\alpha_{k}^{(0)}(t)=\alpha_{k}^{(0)}\left(t_{0}\right) \exp \left\{\int_{t_{0}}^{t}\left(\frac{K(\theta, \theta) \varphi_{k}(\theta)}{\lambda_{k}(\theta)}-\dot{\varphi}_{k}(\theta), \chi_{k}(\theta)\right) d \theta\right\}, k=\overline{1, n}
$$

and, therefore, the solution (15) of the problem will be found uniquely in the space $U$. In this case, the leading term of the asymptotics has the following form:

$$
\begin{aligned}
& y_{\varepsilon 0}(t)=y_{0}^{(0)}(t)+\sum_{k=1}^{n} \alpha_{k}^{(0)}\left(t_{0}\right) e^{\int_{t_{0}}^{t}\left(\frac{K(\theta, \theta) \varphi_{k}(\theta)}{\lambda_{k}(\theta)}-\dot{\varphi}_{k}(\theta), \chi_{k}(\theta)\right) d \theta+\frac{1}{\varepsilon} \int_{t_{0}}^{t} \lambda_{k}(\theta) d \theta} \varphi_{k}(t)+ \\
& +\left(\lambda_{n+1}(t) I-A(t)\right)^{-1} h_{2}(t) e^{\frac{1}{\varepsilon} \int_{t_{0}}^{t} \lambda_{n+1}(\theta) d \theta} \sigma
\end{aligned}
$$

where $y_{0}^{(0)}(t)$ is the solution of the integral system

$$
-A(t) y_{0}(t)-\int_{t_{0}}^{t} K(t, s) y_{0}(s) d s=h_{1}(t) .
$$


Example 1. Let us consider the following problem:

$$
\begin{aligned}
& \left\{\begin{array}{l}
\varepsilon \frac{d y}{d t}=-y+z+\int_{0}^{t} t s \cdot y(s, \varepsilon) d s+2 t+t e^{\frac{i\left(t+t^{3}\right)}{\varepsilon}}, \quad y(0, \varepsilon)=y^{0} \\
\varepsilon \frac{d z}{d t}=-z+\int_{0}^{t}(t-s) \cdot z(s, \varepsilon) d s+t+t^{2} e^{\frac{i\left(t+t^{3}\right)}{\varepsilon}}, z(0, \varepsilon)=z^{0}, t \in[0, T]
\end{array} \Leftrightarrow\right. \\
& \Leftrightarrow \varepsilon \frac{d w}{d t}=A w+\int_{0}^{t} K(t, s) w(s, \varepsilon) d s+H_{1}(t)+H_{2}(t) e^{\frac{i\left(t+t^{3}\right)}{\varepsilon}}, w(0, \varepsilon)=w^{0}
\end{aligned} \Leftrightarrow
$$

where

$$
\begin{aligned}
& A=\left(\begin{array}{cc}
-1 & 1 \\
0 & -2
\end{array}\right), K(t, s)=\left(\begin{array}{cc}
t s & 0 \\
0 & t-s
\end{array}\right), H_{1}(t)=\left(\begin{array}{c}
2 t \\
t
\end{array}\right), \\
& H_{2}(t)=\left(\begin{array}{c}
t \\
t^{2}
\end{array}\right), w=\left(\begin{array}{c}
y(t, \varepsilon) \\
z(t, \varepsilon)
\end{array}\right), w^{0}=\left(\begin{array}{l}
y^{0} \\
z^{0}
\end{array}\right), t \in[0, T] .
\end{aligned}
$$

Let's try to construct its main term of the asymptotic solution. In this system, the matrix is constant and has the following spectrum: $\left\{\lambda_{1}(t), \lambda_{2}(t)\right\}=\{-1,-2\}$, and a rapidly oscillating multiplier with the inhomogeneity $H_{2}(t)$ corresponds to the spectral value $\lambda_{3}(t)=i\left(1+3 t^{2}\right)$. In this case, the eigenvectors of constant matrices $A$ and $A^{*}$ will have the form

$$
\varphi_{1}=\left(\begin{array}{l}
1 \\
0
\end{array}\right), \varphi_{2}=\left(\begin{array}{c}
-1 \\
1
\end{array}\right), \chi_{1}=\left(\begin{array}{l}
1 \\
1
\end{array}\right), \chi_{2}=\left(\begin{array}{l}
0 \\
1
\end{array}\right)
$$

Regularizing problem (23) using the functions

$$
\begin{aligned}
& \tau_{j}=\frac{1}{\varepsilon} \int_{0}^{t} \lambda_{j}(\theta) d \theta, j=\overline{1,3} \Leftrightarrow \tau_{1}=-\frac{t}{\varepsilon}, \tau_{2}=-\frac{2 t}{\varepsilon}, \\
& \tau_{3}=\frac{1}{\varepsilon} \int_{0}^{t}\left(i\left(1+3 \theta^{2}\right)\right) d \theta=\frac{i}{\varepsilon}\left(t+t^{3}\right)
\end{aligned}
$$

we get the following extended problem:

$$
\begin{aligned}
& L_{\varepsilon} \tilde{w}(t, \tau, \varepsilon) \equiv \varepsilon \frac{\partial \tilde{w}}{\partial t}+\lambda_{1} \frac{\partial \tilde{w}}{\partial \tau_{1}}+\lambda_{2} \frac{\partial \tilde{w}}{\partial \tau_{2}}+\lambda_{3}(t) \frac{\partial \tilde{w}}{\partial \tau_{3}}- \\
& -A \tilde{w}-\tilde{J} \tilde{w}=H_{1}(t)+H_{2}(t) e^{\tau_{3}},\left.\tilde{w}(t, \tau, \varepsilon)\right|_{t=0, \tau=(0,0,0)}=w^{0}
\end{aligned}
$$

where $\tau=\left(\tau_{1}, \tau_{2}, \tau_{3}\right), \tilde{J}$ is extension of the integral operator $J$ on series of the form

$$
\tilde{w}(t, \tau, \varepsilon)=\sum_{k=0}^{\infty} \varepsilon^{k} w_{k}(t, \tau)
$$

with coefficients $w_{k}(t, \tau)$ from the space $U$ of vector functions

$$
w(t, \tau)=w_{0}(t)+\sum_{j=1}^{3} w_{j}(t) e^{\tau_{j}}, w_{k}(t) \in C^{\infty}\left([0, T], \mathbb{C}^{2}\right), k=\overline{0,3} .
$$

This extension has the form $\tilde{J} \tilde{w}(t, \tau, \varepsilon)=\sum_{r=0}^{\infty} \varepsilon^{r} \sum_{s=0}^{r} R_{r-s} y_{s}(t, \tau)$, where the operators $R_{v}: U \rightarrow U$ are calculated by the formulas:

$$
\begin{aligned}
& R_{0} w(t, \tau)=\int_{0}^{t} K(t, s) w_{0}(s) d s \\
& R_{1} w(t, \tau)=\sum_{j=1}^{3}\left[\frac{K(t, t) w_{j}(t)}{\lambda_{j}(t)} e^{\tau_{j}}-\frac{K(t, 0) w_{j}(0)}{\lambda_{j}(0)}\right], \ldots
\end{aligned}
$$


(operators $R_{v}$ at $v \geq 2$ we do not write out, because we do not need them when constructing the leading term of the asymptotics). Defining the solution of the problem (24) in the form of series (25), we obtain the following iterative problems:

$$
\begin{gathered}
L w_{0} \equiv \lambda_{1} \frac{\partial w_{0}}{\partial \tau_{1}}+\lambda_{2} \frac{\partial w_{0}}{\partial \tau_{2}}+\lambda_{3}(t) \frac{\partial w_{0}}{\partial \tau_{3}}-A w_{0}-R w_{0}= \\
=H_{1}(t)+H_{2}(t) e^{\tau_{3}},\left.w_{0}(t, \tau)\right|_{t=0, \tau=0}=w^{0} ; \\
L w_{1}=-\frac{\partial w_{0}}{\partial t}+R_{1} w_{0}, w_{1}(0,0)=0 ;
\end{gathered}
$$

The solution of the first iterative problem (26) will be as follows:

$$
w_{0}(t, \tau)=w_{0}(t)+\sum_{k=1}^{2} \alpha_{k}(t) \varphi_{k} e^{\tau_{k}}+\left(\lambda_{3}(t) I-A\right)^{-1} H_{2}(t) e^{\tau_{3}}
$$

where $\alpha_{k}(t) \in C^{\infty}\left(\left[t_{0}, T\right], \mathbb{C}^{2}\right)$ are arbitrary functions, $k=1,2, y_{0}^{(0)}(t)$ is the solution of the integral system

$$
\begin{aligned}
& -A w_{0}(t)-\int_{0}^{t} K(t, s) w_{0}(s) d s=H_{1}(t) \Leftrightarrow \\
& \Leftrightarrow w_{0}(t)=\int_{0}^{t}\left(\begin{array}{cc}
t s & \frac{t-s}{2} \\
0 & \frac{t-s}{2}
\end{array}\right) w_{0}(s) d s+\left(\begin{array}{c}
\frac{5}{2} t \\
\frac{1}{2} t
\end{array}\right) .
\end{aligned}
$$

Subordinate (28) to the initial condition $\left.w_{0}(t, \tau)\right|_{t=0, \tau=0}=w^{0}$. Taking into account the form of vector functions (29) and $H_{2}(t)$, we obtain the equation $\sum_{k=1}^{2} \alpha_{k}(0) \varphi_{k}=w^{0}$. Multiplying this equality scalarly by $\chi_{1}(0)$ and $\chi_{2}(0)$, we find the values $\alpha_{1}(0)=y^{0}+$ $z^{0}, \alpha_{2}(0)=z^{0}$. For a complete calculation of the functions $\alpha_{k}(t)$, we pass to the next iterative problem (27). Taking into account that, under the conditions of solvability (9) of problem (27) only exponentials $e^{\tau_{1}}$ and $e^{\tau_{2}}$ are involved, we keep in its right-hand side only terms depending on these exponentials:

$$
-\frac{\partial}{\partial t} \sum_{k=1}^{2} \alpha_{k}(t) \varphi_{k} e^{\tau_{k}}+\sum_{j=1}^{2} \frac{K(t, t) \varphi_{k}}{\lambda_{j}(t)} \alpha_{k}(t) e^{\tau_{j}}
$$

and subordinate the resulting sum to the orthogonality conditions (9). We will have

$$
-\frac{d \alpha_{k}(t)}{d t}+\left(\frac{K(t, t) \varphi_{k}}{\lambda_{k}(t)}, \chi_{k}\right) \alpha_{k}(t)=0, k=1,2
$$

or (in more detail, taking into account the eigenvectors $\varphi_{j}, \chi_{j}$ and the kernel $K(t, t)$ ):

$$
\begin{aligned}
& -\dot{\alpha}_{1}(t)-\left(\left(\begin{array}{cc}
t^{2} & 0 \\
0 & 0
\end{array}\right)\left(\begin{array}{l}
1 \\
0
\end{array}\right),\left(\begin{array}{l}
1 \\
1
\end{array}\right)\right) \alpha_{1}(t)=0, \\
& -\dot{\alpha}_{2}(t)-\frac{1}{2}\left(\left(\begin{array}{cc}
t^{2} & 0 \\
0 & 0
\end{array}\right)\left(\begin{array}{c}
-1 \\
1
\end{array}\right),\left(\begin{array}{c}
0 \\
1
\end{array}\right)\right) \alpha_{2}(t)=0 \quad \Leftrightarrow \dot{\alpha}_{1}(t)=-t^{2} \alpha_{1}(t), \dot{\alpha}_{2}(t)=0 \text {. }
\end{aligned}
$$

Adding to these equations the initial conditions $\alpha_{1}(0)=y^{0}+z^{0}, \alpha_{2}(0)=z^{0}$, found earlier, we uniquely find the functions $\alpha_{1}(t)=e^{-\frac{t^{3}}{3}}\left(y^{0}+z^{0}\right), \alpha_{2}(t)=z^{0}$, and hence, we will uniquely construct solution (28) of the first iterative problem (26). Making a narrowing in it at $\tau_{1}=-\frac{t}{\varepsilon}, \tau_{2}=-\frac{2 t}{\varepsilon}, \tau_{3}=\frac{i}{\varepsilon}\left(t+t^{3}\right)$, we obtain the leading term of the asymptotic solution of the problem (23):

$$
w_{\varepsilon 0}(t)=w_{0}(t)+e^{-\frac{t^{3}}{3}}\left(y^{0}+z^{0}\right)\left(\begin{array}{l}
1 \\
0
\end{array}\right) e^{-\frac{t}{\varepsilon}}+z^{0}\left(\begin{array}{c}
-1 \\
1
\end{array}\right) e^{-\frac{2 t}{\varepsilon}}+\left(\begin{array}{c}
\frac{t\left(3 i t^{2}+2+i+t\right)}{\left(3 i t^{2}+1+i\right)\left(3 i t^{2}+2+i\right)} \\
\frac{t^{2}}{3 i t^{2}+2+i}
\end{array}\right) e^{\frac{i}{\varepsilon}\left(t+t^{3}\right)}
$$


where $w_{0}(t)$ is the solution of the integral system (29). It is seen from (30) that, at the exact solution $w(t, \varepsilon)$ of the problem (23) does not tend to the solution $w_{0}(t)$ of the integral system (29) at $\varepsilon \rightarrow+0$, but performs quick oscillations near it.

\section{Conclusions}

From the expression (22b) for $y_{\varepsilon 0}(t)$, it can be seen that the construction of the leading term of the asymptotics of the solution to problem (1) is significantly influenced by both the rapidly oscillating inhomogeneity and the kernel of the integral operator.

Note that the application of other asymptotic methods (for example, the method of boundary functions [17-19]) to problems of type (1) with rapidly oscillating inhomogeneities is problematic, since many of them rely heavily on the fact that all points of the spectrum (including the spectral value of the inhomogeneity) lie in the open half-plane $\operatorname{Re} \lambda<0$.

Author Contributions: The contribution of each author is equal. All authors have read and agreed to the published version of the manuscript.

Funding: This work was supported by grant No. AP05133858 of the Ministry of Education and Science of the Republic of Kazakhstan.

Institutional Review Board Statement: Not applicable.

Informed Consent Statement: Not applicable.

Data Availability Statement: Not applicable.

Conflicts of Interest: The authors declare no conflict of interest.

\section{References}

1. Feschenko, S.F.; Shkil, N.I.; Nikolenko, L.D. Asymptotic Methods in the Theory of Linear Differential Equations; Naukova Dumka: Kiev, Ukraine, 1966.

2. Daletsky, Y.L. The asymptotic method for some differential equations with oscillating coefficients. DAN USSR 1962, 143, 1026-1029.

3. Lomov, S.A. Introduction to General Theory of Singular Perturbations; Vol. 112 of Translations of Math; Monographs, American Mathematical Society: Providence, RI, USA, 1992.

4. Lomov, S.A.; Lomov, I.S. Foundations of Mathematical Theory of Boundary Layer; Publishing House of Moscow State University: Moscow, Russia, 2011.

5. Ryzhih, A.D. Asymptotic solution of a linear differential equation with a rapidly oscillating coefficient. Trudy MEI 1978, 357, 92-94.

6. Kalimbetov, B.T.; Temirbekov, M.A.; Khabibullaev, Z.O. Asymptotic solution of singular perturbed problems with an instable spectrum of the limiting operator. Abstr. Appl. Anal. 2012, 2012, 120192. [CrossRef]

7. Imanbaev, N.S.; Kalimbetov, B.T.; Temirbekov, M.A. Asymptotics of solutions of singularly perturbed integro- differential equation with rapidly decreasing kernel. Bull. Ksu Math. 2013, 72, 55-63.

8. Bobodzhanov, A.A.; Safonov, V.F. Singularly perturbed nonlinear integro-differential systems with rapidly varying kernels. Math. Notes 2002, 72, 605-614. [CrossRef]

9. Bobodzhanov, A.A.; Safonov, V.F. Singularly perturbed integro-differential equations with diagonal degeneration of the kernel in reverse time. J. Differ. Equat. 2004, 40, 120-127. [CrossRef]

10. Bobodzhanov, A.A.; Safonov, V.F. Asymptotic analysis of integro-differential systems with an unstable spectral value of the integral operator's kernel. Comput. Math. Math. Phys. 2007, 47, 65-79. [CrossRef]

11. Bobodzhanov, A.; Safonov, V.; Kachalov, V.I. Asymptotic and pseudoholomorphic solutions of singularly perturbed differential and integral equations in the lomov's regularization method. Axioms 2019, 8, 27. [CrossRef]

12. Bobodzhanov, A.A.; Safonov, V.F. A problem with inverse time for a singularly perturbed integro-differential equation with diagonal degeneration of the kernel of high order. Izv. Math. 2016, 80, 285-298. [CrossRef]

13. Bobodzhanov, A.A.; Kalimbetov, B.T.; Safonov, V.F. Integro-differential problem about parametric amplification and its asymptotical integration. Int. J. Appl. Math. 2020, 33, 331-353. [CrossRef]

14. Omuraliev, A.S. Asymptotics of solving a system of linear equations of parabolic type with a small parameter. Equ. J. Differ. Equat. 2019, 55, 878-882. [CrossRef]

15. Kalimbetov, B.T.; Safonov, V.F. Regularization method for singularly perturbed integro-differential equations with rapidly oscillating coefficients and with rapidly changing kernels. Axioms 2020, 9, 131. [CrossRef] 
16. Safonov, V.F.; Bobodzhanov, A.A. Course of Higher Mathematics. Singularly Perturbed Equations and the Regularization Method: Textbook; Publishing House of MPEI: Moscow, Russia, 2012.

17. Vasil'yeva, A. B.; Butuzov, V.F. Asymptotic Methods in the Theory of Singular Perturbations; Vysshaya Shkola: Moscow, Russia, 1990.

18. Imanaliev, M. Asymptotic Methods in the Theory of Singularly Perturbed Integro-Differential Equations; Ilim: Frunze, Kyrgyzstan, 1972.

19. Dauylbaev, M.K. The asymptotic behavior of solutions to singularly perturbed nonlinear integro-differential equations. Sib. Math. J. 2000, 41, 49-60. [CrossRef] 